\title{
Intolerable wide and narrow QRS complex tachycardia in the same patient: What is your diagnosis? is
}

\author{
Tolga Aksu *, Tümer Erdem Güler, Kazım Serhan Özcan \\ Department of Cardiology, Kocaeli Derince Education and Research Hospital,Derince, Kocaeli, Turkey
}

\section{A R T I C L E I N F O}

\section{Article history:}

Received 6 March 2016

Received in revised form 23 March 2016

Accepted 25 March 2016

Available online 11 April 2016

\section{Keywords:}

Ablation

AVNRT

Electrophysiology

Supraventricular tachycardia

\begin{abstract}
A B S T R A C T
A 54-year-old female with recurrent syncope was referred to our institution for electrophysiological study. The patient had two documented intolerable tachycardia; one of them was a wide QRS, and the other one was a narrow QRS. As a clinical puzzle format, we aimed to present our basic electrophysiological approach of an intolerable and rare clinical tachycardia.

(C) 2016 The Society of Cardiovascular Academy. Production and hosting by Elsevier B.V. All rights reserved. This is an open access article under the CC BY-NC-ND license (http://creativecommons.org/licenses/by-nc-nd/4.0/).
\end{abstract}

\section{Introduction}

Common type atrioventricular nodal re-entrant tachycardia (AVNRT) and atrioventricular re-entrant tachycardia (AVRT) via an accessory pathway are the most common causes of sustained regular narrow complex tachycardia, the electrocardiographic presentation of the tachycardia may be rarely with wide QRS. ${ }^{1}$ Distinguishing between these arrhythmias can often be challenging during electrophysiology study (EPS). The detection of a wide QRS tachycardia switching to a narrow QRS tachycardia is an uncommon clinical presentation., ${ }^{2,3}$

\section{Case question}

A 54-year-old female with recurrent syncope and palpitation attack was referred to our institution for EPS. The patient had two documented tachycardia; one of them is a wide QRS left bundle branch block and the other one is a narrow QRS regular tachycardia (Fig. 1). Both tachycardias terminated with administration of intravenous diltiazem. First, a sustained tachycardia with wide QRS was induced by programmed atrial stimulation (PAS) (Fig. 2A and B). Due to hemodynamic deterioration, we were forced to terminate tachycardia in a short time. Surprisingly, the tachycardia could be terminated by atrial burst pacing. We tried to induce the tachycardia again for differential diagnosis. The tachycardia was not induced by programmed by ventricular pacing. However, PAS with same protocol induced two distinct narrow QRS

\footnotetext{
ts Peer review under responsibility of The Society of Cardiovascular Academy.

* Corresponding author. Tel.: +90 5319903278; fax: + 902623178000.

E-mail address: aksutolga@gmail.com (T. Aksu).
}

tachycardia (Fig. 2C and D). Dual atrioventricular (AV) node physiology and a single AV nodal echo beat were demonstrated. Ventricular pacing during sinus rhythm showed ventriculoatrial (VA) conduction that was concentric and detrimental. The induction of the tachycardia by atrial extrastimulus testing and the response to a single premature atrial extrastimulus introduced during His refractoriness were repeatedly observed. Due to significant hemodynamic effects of the tachycardia, all pacing maneuvers could not be performed during the tachycardia. On the base of the present findings, what are the mechanisms of the tachycardia?

\section{Case answer}

The presence of 1:1 VA relationship during wide QRS tachycardia (Fig. 2A) and the termination of the tachycardia by atrial stimulation are unlikely for ventricular tachycardia. Due to short VA interval, the most likely mechanisms for the wide QRS tachycardia likely are an antidromic atriofascicular bypass tract tachycardia, typical AVNRT, or atrial tachycardia with aberrant conduction. However, the transition of a narrow QRS tachycardia to a wide (Fig. 2B) without a change in cycle length and in VA interval excludes the presence of an atriofascicular bypass tract or antidromic AV reentrant tachycardia. Also, constant HV intervals at the transition from narrow QRS to wide QRS tachycardia and typical LBBB pattern during the wide QRS tachycardia indicate that the mechanism of the wide QRS tachycardia is a supraventricular tachycardia with aberrant conduction. The concentric atrial activation pattern and short septal VA interval in the wide QRS tachycardia and one of the narrow QRS tachycardia favor typical AVNRT. The tachycardia with 1:1 AV relationship shown in Fig. 2B was terminated with AV block, indicating that the tachycardia was dependent on the AV node, also favors AVNRT. 


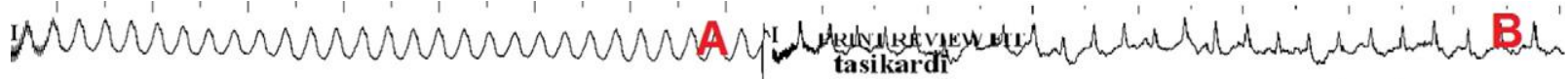

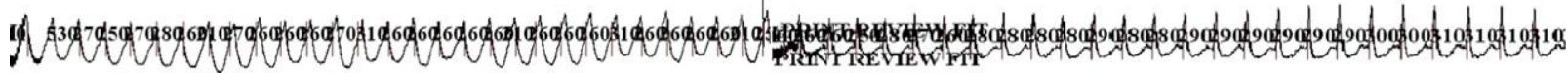
PRINT REVIEW FIT

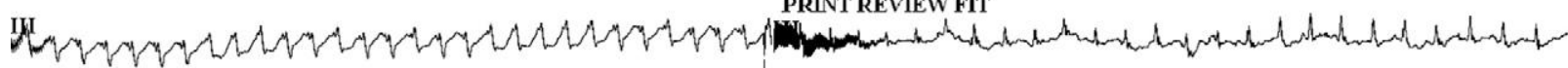

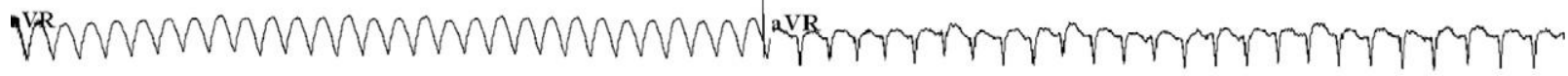

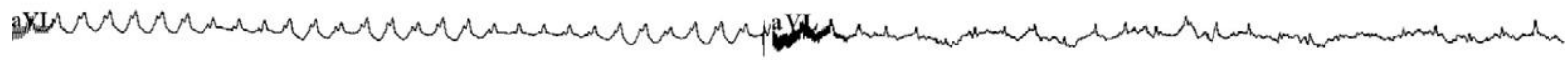

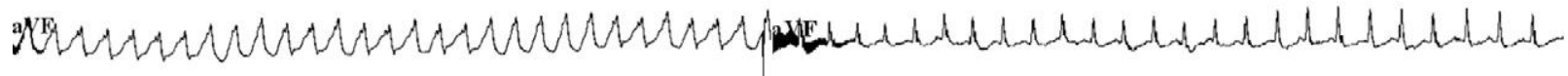

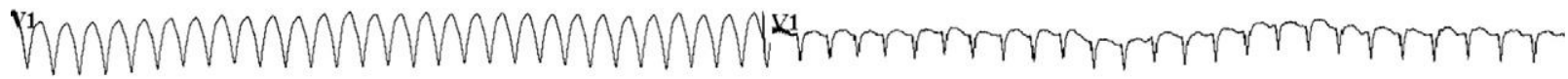

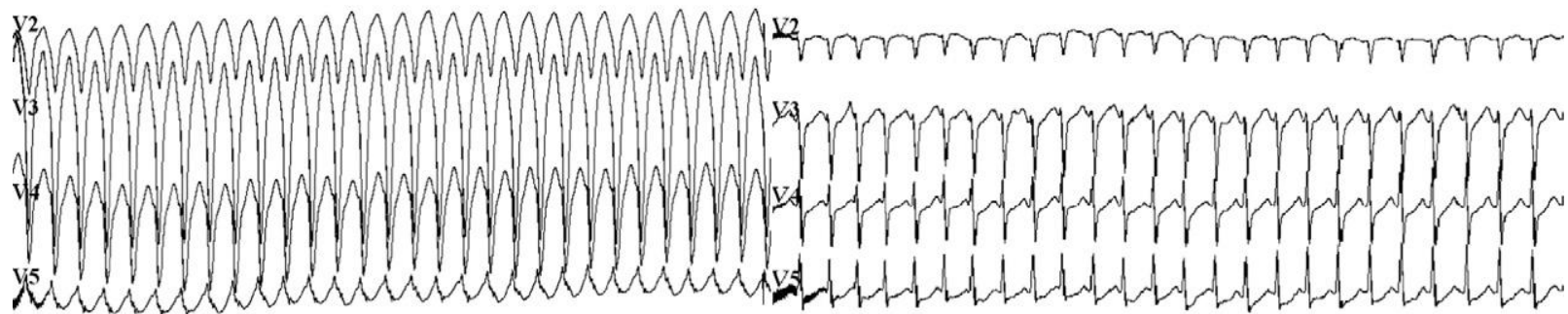

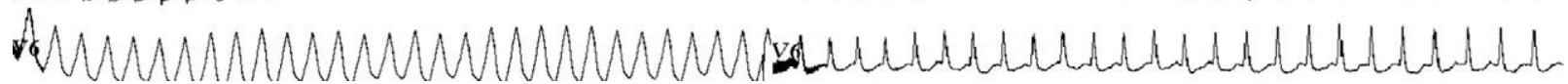

Fig. 1. (A) Twelve-lead electrocardiogram of the clinical wide QRS tachycardia. (B) Twelve-lead electrocardiogram of the clinical narrow QRS tachycardia.

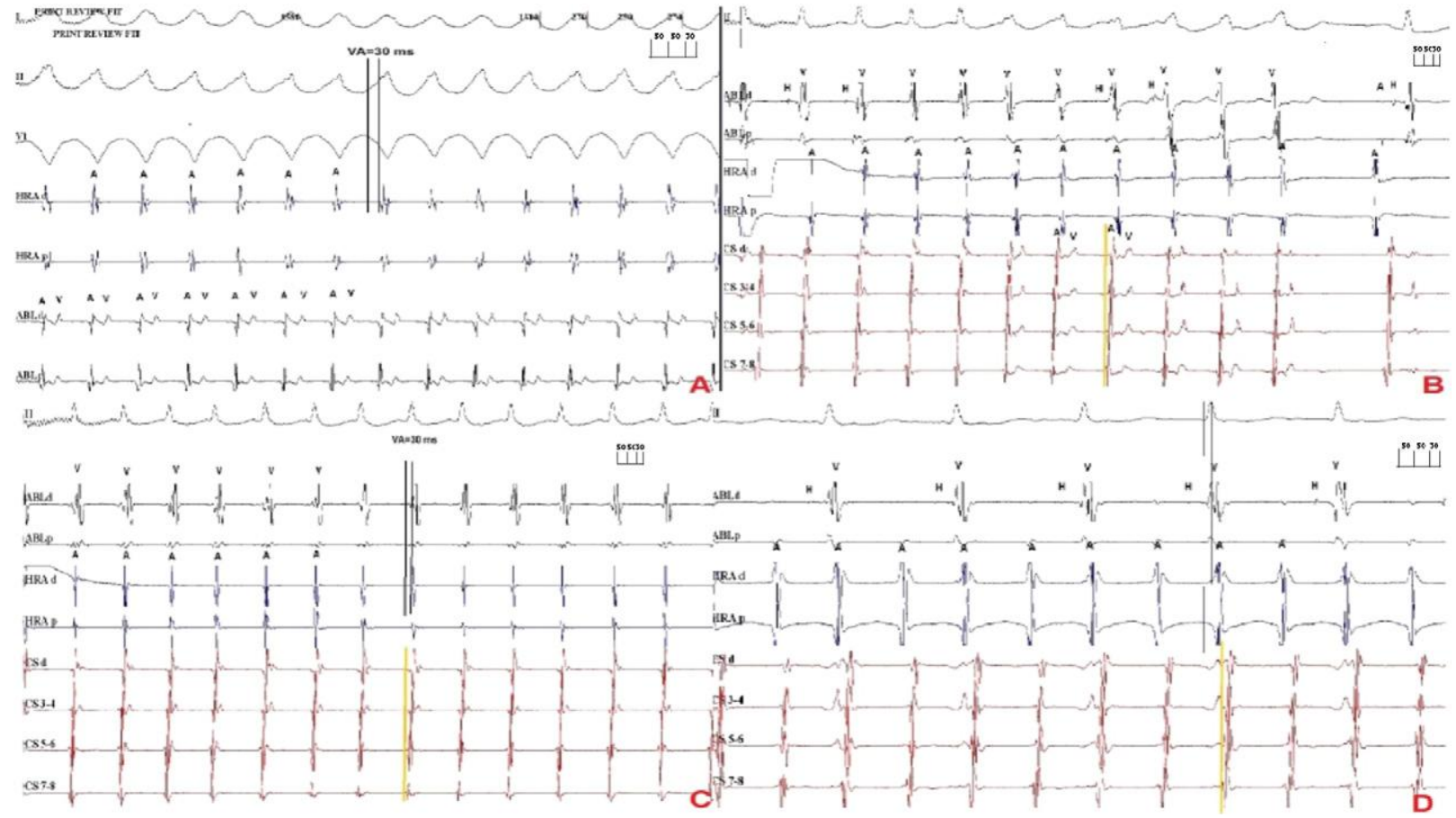

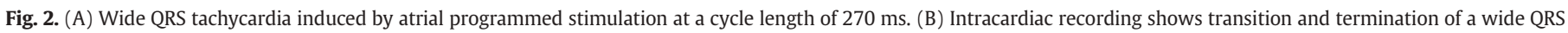

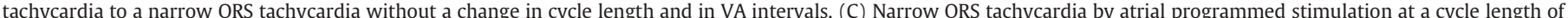

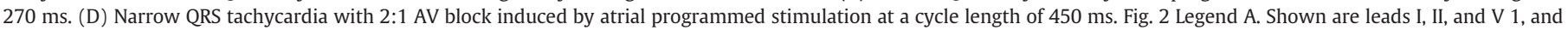

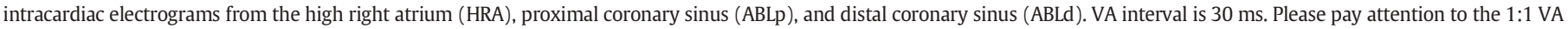

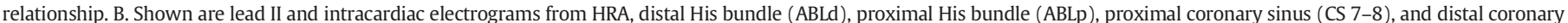

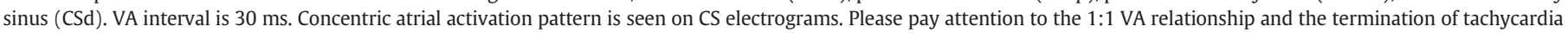

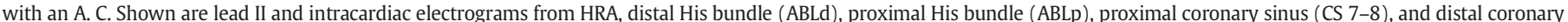

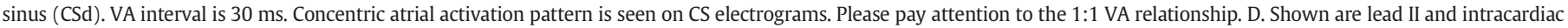

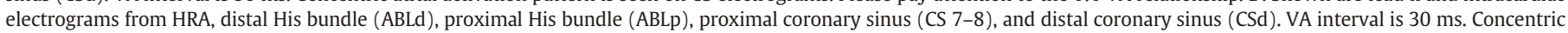
atrial activation pattern is seen on CS electrograms. 
The presence of constant VA and AV interval rules atrial tachycardia with aberrant conduction out. Also, the termination of the tachycardia with an A makes atrial tachycardia unlikely. For the second tachycardia, which is a narrow QRS tachycardia (Fig. 2C) with 1:1 AV relationship, the differential diagnosis mainly includes septal atrial tachycardia, typical AVNRT, and automatic junctional tachycardia (JT) due to short VA interval and concentric atrial activation pattern. The presence of constant VA and AV interval are unlikely for atrial tachycardia. Also, dual AV node physiology and very short VA interval favor but are not confirmatory of typical AVNRT. The reproducible induction of the tachycardia with premature stimulation is unusual in automatic JT. The delay of the subsequent tachycardia beat after his refractory atrial extrastimulus rules JT out. For the last tachycardia with 2:1 AV block (Fig. 2D), the differential diagnosis mainly includes atrial tachycardia and typical AVNRT due to concentric atrial activation pattern. In this tachycardia, a different atrial activation pattern with the early atrial activation at the HRA and His bundle region was detected. Although the proximal to distal CS activation seems as concentric, the early activation at the HRA and his bundle should suggest the tachycardia may be atrial tachycardia with an eccentric atrial activation and AV block. The VA interval of $30 \mathrm{~ms}$ is too short for an atrial tachycardia. Although the constant VA relation could not exclude atrial tachycardia in every time, we accepted typical AVNRT as the responsible mechanism of all seen tachycardia. Thus, we decided to ablate slow pathway. Any tachycardia could not be further induced after modification of the slow pathway.

\section{Discussion}

AVNRT is a relatively common supraventricular arrhythmia, and an accurate diagnosis is essential before proceeding with ablative therapy. Along with specific electrocardiographic clues, specific electrophysiological techniques enable accurate diagnosis of AVNRT. Para-Hisian pacing, "His" refractory extrasystole and entrainment techniques are crucial to differentiate AVNRT from other supraventricular tachycardias, especially orthodromic reciprocal tachycardia with a concealed septal accessory pathway. ${ }^{4}$ In this case presentation, we could not perform these discrimination techniques due to hemodynamic effects of the tachycardia. However, we tried to explain that the discrimination of AVNRT can be done with basic electrophysiological properties without pacing maneuvers. Also, to the best of our knowledge, we firstly presented all tachycardia forms of typical AVNRT in a same patient.

\section{References}

1. Morady F. Catheter ablation of supraventricular arrhythmias: state of the art. Pacing Clin Electrophysiol 2004;27:125-142.

2. Uetake S, Maruyama M, Yamamoto T, Hata N, Seino Y, Shimizu W. Conversion from two types of wide QRS complex tachycardia to narrow QRS complex tachycardia: what are the mechanisms? J Cardiovasc Electrophysiol 2016;27:129-130.

3. Bohora S, Wide QRS. Tachycardia changing to a narrow QRS tachycardia. What is the mechanism? Heart Rhythm 2015;12:1698-1700.

4. Katritsis DG, Camm AJ. Atrioventricular nodal reentrant tachycardia. Circulation 2010:122:831-840. 